Review

\title{
Menahydroquinone-4 Prodrug: A Promising Candidate Anti-Hepatocellular Carcinoma Agent
}

\author{
Munechika Enjoji ${ }^{\dagger} *$, Daisuke Watase ${ }^{\dagger}$, Kazuhisa Matsunaga, Mariko Kusuda, \\ Nami Nagata-Akaho, Yoshiharu Karube and Jiro Takata ${ }^{\dagger}$
}

Faculty of Pharmaceutical Sciences, Fukuoka University, 8-19-1 Nanakuma, Jonan-ku, Fukuoka 814-180, Japan; E-Mails: watase@adm.fukuoka-u.ac.jp (D.W.); k-matsu@fukuoka-u.ac.jp (K.M.); kusuda@adm.fukuoka-u.ac.jp (M.K.); akaho@adm.fukuoka-u.ac.jp (N.N.-A.); karube@adm.fukuoka-u.ac.jp (Y.K.); jtakata@fukuoka-u.ac.jp (J.T.)

$\dagger$ These authors contributed equally to this work.

* Author to whom correspondence should be addressed; E-Mail: enjoji@adm.fukuoka-u.ac.jp; Tel.: +81-92-871-6631 (ext. 6694); Fax: +81-92-863-0389.

Academic Editor: Stephen L. Chan

Received: 20 May 2015 / Accepted: 13 July 2015 / Published: 22 July 2015

\begin{abstract}
Recently, new therapeutics have been developed for hepatocellular carcinoma (HCC). However, the overall survival rate of HCC patients is still unsatisfactory; one of the reasons for this is the high frequency of recurrence after radical treatment. Consequently, to improve prognosis, it will be important to develop a novel anti-tumor agent that is especially effective against HCC recurrence. For clinical application, long-term safety, together with high anti-tumor efficacy, is desirable. Recent studies have proposed menahydroquinone-4 1,4-bis- $N, N$-dimethylglycinate hydrochloride (MKH-DMG), a prodrug of menahydroquinone$4(\mathrm{MKH})$, as a promising candidate for HCC treatment including the inhibition of recurrence; MKH-DMG has been shown to achieve good selective accumulation of MKH in tumor cells, resulting in satisfactory inhibition of cell proliferation in des- $\gamma$-carboxyl prothrombin (DCP)positive and DCP-negative HCC cell lines. In a spleen-liver metastasis mouse model, MKH-DMG has been demonstrated to have anti-proliferation and anti-metastatic effects in vivo. The characteristics of MKH-DMG as a novel anti-HCC agent are presented in this review article.
\end{abstract}


Keywords: vitamin K; hepatocellular carcinoma; anti-cancer drug; drug delivery; menaquinone-4

\section{Introduction}

Intracellular levels of vitamin $\mathrm{K}$ and its homologs are significantly lower in most hepatocellular carcinomas (HCCs) as compared with background non-tumor areas [1]; vitamin K-dependent carboxylation reactions are impaired in HCC cells. Vitamin K can inhibit the growth of HCC cells in a dose-dependent manner. However, vitamin $\mathrm{K}$ uptake is lower in HCC cells relative to normal hepatocytes in vitro [2]. These findings support the hypothesis that differences in the ability to absorb vitamin $\mathrm{K}$ lead to the individual sensitivity of $\mathrm{HCC}$ to vitamin $\mathrm{K}$. Nowadays, the vitamin $\mathrm{K}_{2}$ (menaquinone) homolog menaquinone-4 (MK-4) is used for the treatment of osteoporosis, and its long-term safety has been confirmed [3-6]. There is a possibility that MK-4 can suppress the progression of HCC [7-12]. In some small-scale studies, MK-4 treatment has been reported to reduce the onset of $\mathrm{HCC}$ in patients with liver cirrhosis and $\mathrm{HCC}$ recurrence after curative surgical resection or radiofrequency ablation $[13,14]$. Indeed, MK-4 is expected to inhibit de novo carcinogenesis, HCC proliferation and HCC recurrence with long-term safety. However, no significant inhibiting effect was proven in a large-scale, double-blind and randomized control study [15]. A meta-analysis of randomized controlled trials, failed to confirm significantly better tumor recurrence-free survival at one year, and there was no beneficial effect on the overall survival [16].

Because the anti-HCC effect of MK-4 may be dependent on the delivery of its metabolite, menahydroquinone-4 (MKH), it is hypothesized that effective delivery of MKH to HCC cells leads to inhibition of HCC proliferation, metastasis and recurrence. However, MKH itself has easily oxidizable characteristics and is unsuitable for clinical use. In our previous studies, menahydroquinone-4 1,4-bis- $N, N$-dimethylglycinate hydrochloride (MKH-DMG), the ester derivative of $\mathrm{MKH}$, showed excellent MKH delivery potential regarding the liver without the reductive activation process of MK-4 to $\mathrm{MKH}[17,18]$. To improve the prognosis of patients with HCC, an agent with high anti-tumor effectiveness and a good safety profile needs to be developed. Recent studies suggest that MKH-DMG has promising anti-HCC characteristics, such as intracellular MKH delivery and inhibition of tumor progression [19].

\section{MKH Delivery System in HCC Cells}

The schema for the conversion process associated with the MKH delivery system in HCC cells is shown in Figure 1. Des- $\gamma$-carboxy prothrombin (DCP) also known as the protein induced by vitamin $\mathrm{K}$ absence-II (PIVKA-II), an abnormal prothrombin with incomplete carboxylation, is a HCC-specific tumor marker [20,21]. Additionally, DCP functions as a predictive factor for vascular invasion, metastasis and tumor progression; it is associated with the poor prognosis of HCC patients [22-27]. The vitamin $\mathrm{K}$ content in HCC cells has the ability to restrict DCP production [1,28-30]. MKH, a fully reduced form of MK-4, is a cofactor of $\gamma$-glutamyl carboxylase (GGCX) that converts glutamate residue into the $\gamma$-carboxyglutamate residue of vitamin $\mathrm{K}$-dependent proteins such as prothrombin [31-33]. 
Therefore, ancillary to vitamin K-dependent carboxylation, $\mathrm{MKH}$ is stoichiometrically converted into menaquinone-4 epoxide (MKO). Utilization of $\mathrm{MKH}$ is restricted in $\mathrm{HCC}$ tissue. Because $\mathrm{MKH}$ availability regulates the rate of carboxylation [34], reduction of MKH availability in HCC cells may result in the increase in DCP.

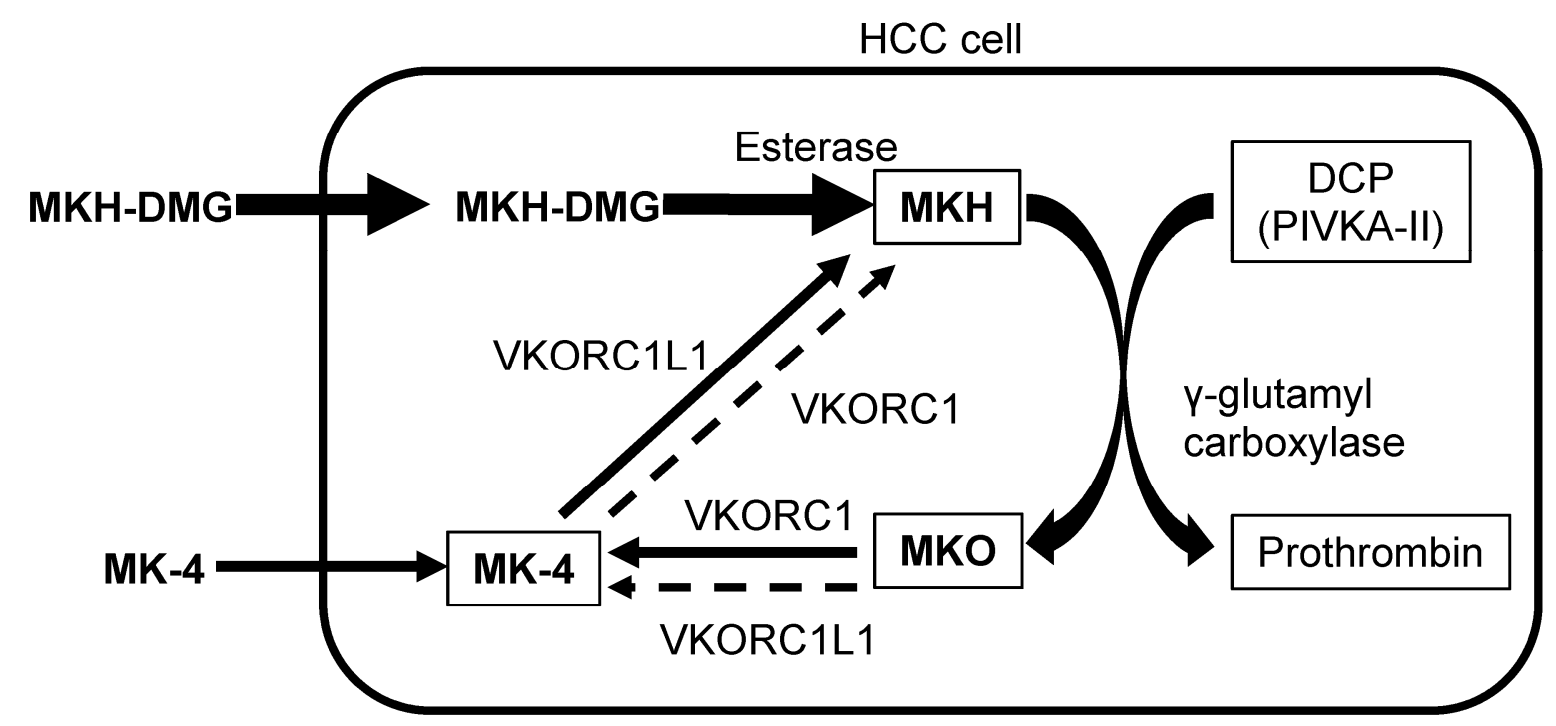

Figure 1. Schema of MKH delivery system in HCC cells. MKH-DMG, menahydroquinone-4 1,4bis- $N, N$-dimethylglycinate hydrochloride; MK-4, menaquinone-4; $\mathrm{MKH}$, menahydroquinone-4; MKO, menaquinone-4 epoxide; DCP, des- $\gamma$-carboxyl prothrombin; PIVKA-II, protein induced by vitamin $\mathrm{K}$ absence-II; VKORC1, vitamin $\mathrm{K}$ 2,3-epoxide reductase complex subunit 1; VKORC1L1, VKORC1 like-1; HCC, hepatocellular carcinoma.

MKH-DMG, an MKH prodrug, is hydrolyzed into MKH by esterase after its uptake by cells. GGCX is required for its activity and depends upon $\mathrm{MKH}$, which is generated mainly by vitamin $\mathrm{K}$ 2,3-epoxide reductase complex, subunit 1-like 1 (VKORC1L1). VKORC1L1 promotes the reduction of MK-4 to $\mathrm{MKH}$ and supports vitamin $\mathrm{K}$ hydroquinone-mediated intracellular antioxidation, which is critical for cell survival [35]. In our previous studies, MKH-DMG effectively supplied MKH to HCC cells without reductive activation $[17,18]$.

\section{Inhibition of HCC Cell Proliferation by MKH-DMG}

MKH-DMG has an inhibitory effect regarding cell proliferation in HCC cell lines in vitro. In our cell viability assay, MKH-DMG suppressed the proliferation of HCC cells in a time- and dose-dependent manner both in DCP-positive (PLC/PRF/5 and Hep3B) and DCP-negative (SK-Hep1) cell lines [19]. $\mathrm{A}$ rapid and intensive suppression effect was evident at $48 \mathrm{~h}$ after MKH-DMG administration at a concentration of $20 \mu \mathrm{M}$ (Table 1). In contrast, the effect of MK-4 was clearly weaker and the suppression of cell proliferation was not distinct until $72 \mathrm{~h}$ at a concentration of $\geq 40 \mu \mathrm{M}$ (Table 1). MKH-DMG showed significantly lower IC 50 values $(14-37 \mu \mathrm{M})$ and exhibited 4-18 times stronger proliferationsuppressing activity than MK-4. When cell injury was estimated by LDH release into the medium, no indication of injury was evident during MKH-DMG treatment. MK-4 is usually prescribed at a dose of $45 \mathrm{mg} /$ day (three times per day) with a good long-term safety profile in the treatment of osteoporosis 
patients. At this dose, the maximum plasma concentration is $1 \mu \mathrm{M}$ [36]; in a previous distribution study of oral MK-4 administration, the hepatic concentration was $>10$ times higher than the plasma concentration [37]. These results indicate that the IC50 values of MKH-DMG used in the treatment of HCC cell lines may be clinically reasonable levels, and that MKH-DMG is potentially a promising anti-HCC agent available for safe clinical use.

Table 1. Effects for HCC cells (Reference [19]).

\begin{tabular}{ccccc}
\hline Treatment & Inhibition of Cell & MKH Delivery & Downregulation of & HCC inhibition \\
& Proliferation (in vitro) & (in vitro) & DCP $($ in vitro $)$ & (in vivo) \\
\hline MKH-DMG (concentration) & obvious $(20 \mu \mathrm{mol} / \mathrm{L})$ & obvious $(25 \mu \mathrm{mol} / \mathrm{L})$ & obvious $(10 \mu \mathrm{mol} / \mathrm{L})$ & obvious $(0.2 \mu \mathrm{mol} / \mathrm{day})$ \\
MK-4 (concentration) & weak $(40 \mu \mathrm{mol} / \mathrm{L})$ & weak $(25 \mu \mathrm{mol} / \mathrm{L})$ & obvious $(10 \mu \mathrm{mol} / \mathrm{L})$ & - \\
\hline
\end{tabular}

* MKH-DMG was dissolved in drinking water $(40 \mu \mathrm{mol} / \mathrm{L})$ and provided ad libitum.

\section{Effective MKH Delivery into HCC Cells by MKH-DMG}

Inhibition of HCC proliferation, metastasis and recurrence by MKH-DMG and MK-4 are determined by their ability to deliver MKH to HCC cells. It is difficult to measure MKH levels accurately in HCC cells because of its highly oxidative characteristics regarding MK-4. However, MKO levels can be substituted for MKH levels (Figure 1). MKH delivery via MKH-DMG or MK-4 (both at a concentration of $25 \mu \mathrm{M}$ ) to HCC cell lines has been estimated by means of intracellular MKO and MK-4 levels [19]. MKH-DMG treatment induced a rapid and time-dependent increase in intracellular MKO and MK-4 levels (Table 1). In this case, MK-4 is an oxidation product of MKH (Figure 1). MKH-DMG is taken up by HCC cells and effectively converted into MKH. Conversely, MK-4 administration did not lead to an increase in MKO and MK-4 levels (Table 1), which supports the evidence that the MK-4 uptake rate was lower in HCC cells relative to normal hepatocytes [2]. When the AUC of the intracellular concentration versus time profile was determined, the $\mathrm{AUC}_{0-72} \mathrm{~h}$ values for $\mathrm{MKH}$ were 3.5-15 times higher after MKH-DMG treatment than those after MK-4 treatment, regardless of whether DCP-positive or DCP-negative cell lines were used. These results indicate that MKH-DMG administration is an effective method for the delivery of MKH to HCC cells.

MKH-DMG as an MKH prodrug shows satisfactory cell-membrane permeability and is effectively hydrolyzed into $\mathrm{MKH}$ by esterase present in $\mathrm{HCC}$ cells, leading to the rapid and intensive inhibition for HCC proliferation. Perhaps the uptake process for MKH-DMG is different to that for MK-4. Investigation of MKH-DMG uptake including transporter system may be an important target for future research.

\section{Downregulation of DCP by MKH-DMG}

In a recent investigation, DCP/PIVKA-II was reported to act as a growth and metastasis factor for HCC, and to exacerbate its prognosis [27]. Therefore, depression of DCP may be a prospective target for developing a novel treatment against DCP-positive HCC. This therapeutic strategy is applicable to MKH-DMG administration, which can achieve sufficiently high MKH delivery. The effect of MKH-DMG regarding the DCP level in a DCP-positive PLC/PRF/5 cell line was evaluated by measuring the DCP concentration in culture media at $72 \mathrm{~h}$ after treatment [19]. DCP levels had clearly decreased after 
MKH-DMG $(10 \mu \mathrm{M})$ and MK-4 $(10 \mu \mathrm{M})$ treatments $(2.0 \pm 0.0 \mathrm{mAU} / \mathrm{mL}$, and $1.3 \pm 0.6 \mathrm{mAU} / \mathrm{mL}$, respectively) relative to untreated controls $(43 \pm 3.6 \mathrm{mAU} / \mathrm{mL})$. However, as described above, cell proliferation was only suppressed after MKH-DMG treatment and not after MK-4 treatment. Because growth inhibition caused by MKH-DMG has also been demonstrated in DCP-negative HCC cells, DCP suppression may not be important for MKH-DMG and MKH regarding the inhibition of HCC growth. Other vitamin K-dependent proteins, with the exception of DCP, may be critical regarding the effect of MKH-DMG, and such vitamin K-dependent proteins should be explored.

\section{Induction of Cell-Cycle Arrest by MKH-DMG}

The underlying mechanism concerning the effect of MKH-DMG on HCC cell growth suppression should be investigated. Generally, cell-cycle arrest has been considered to be primarily associated with the anti-proliferative action of MK-4 [7-9,11,37,38]. Aberrant expression of NF- $\mathrm{BB}$ is linked to cyclin D1 [10], and to the onset and progression of HCC tumorigenesis [38]. Because both cyclin D1 and $\mathrm{NF}-\kappa \mathrm{B}$ are associated with cellular migration $[39,40]$, downregulation of NF- $\kappa \mathrm{B}$ and cyclin D1 by MKH-DMG treatment may contribute to the inhibition of HCC cell growth and invasion.

In our analysis, it is suggested that $\mathrm{G}_{1} / \mathrm{S}$ arrest is one of the mechanisms involved in the inhibition of HCC cell proliferation induced by MKH-DMG [19]. Flow cytometric analysis of MKH-DMG-treated $\mathrm{PLC} / \mathrm{PRF} / 5$ cells in vitro revealed an increase in $\mathrm{G}_{1}$ phase cells and a decrease in $\mathrm{S}$ phase cells. In Western blot analysis, the expression of cell cycle-related proteins (cyclin D1, cyclin D3 and CDK4) decreased and was completely suppressed after $48 \mathrm{~h}$ of MKH-DMG treatment in both DCP-positive (PLC/PRF/5 and Hep3B) and DCP-negative (SK-Hep-1) HCC cell lines. Conversely, similar treatment using MK-4 induced only a slight decrease in the levels of these proteins. Additionally, NF- $\kappa \mathrm{B}$ was downregulated by MKH-DMG treatment in these HCC cell lines, which might have resulted from effective MKH delivery to the tumor cells using MKH-DMG.

\section{Anti-Proliferation Effect of MKH-DMG in Vivo}

The clinical benefit of MKH-DMG treatment has been demonstrated using xenografted human HCC cells in vivo [19]. In a preceding pharmacokinetic study after oral administration of MKH-DMG, MKH-DMG was found to be delivered to and absorbed by hepatocytes in the same esterized form, and then converted to $\mathrm{MKH}$ in vivo. Using this $\mathrm{MKH}$ delivery system, the effects of MKH-DMG in combating hepatic metastasis and the proliferation of HCC cells (PLC/PRF/5) were examined in a mouse spleen-liver metastasis model (nu/nu mice). Macroscopic findings of liver tumors were estimated at 50 days after transplantation of PLC/PRF/5 cells in MKH-DMG treated and untreated groups. Increases in both liver weight and the percentage of cancer surface area were significantly suppressed in the MKHDMG treatment group as compared with the untreated group. In the MKH-DMG treatment group, plasma DCP, which is produced by HCC cells, was not detected, although liver metastasis could not be suppressed completely. These findings mean that MKH is definitely delivered to the metastasized HCC cells and that DCP is suppressed by MKH; they suggest the possibility that MKH-DMG could function as an anti-HCC agent in humans. However, subsequent investigation is required to show whether or not a similar effect can be demonstrated after MKH-DMG treatment of human HCC. 


\section{Conclusions}

The effects of MKH-DMG and MK-4 regarding HCC cells are summarized in Table 1. Reduced uptake of therapeutic agents by cancer cells is one of the important factors affecting resistance to chemotherapy. Consequently, the development of compounds with tumor-specific cell penetration properties is still a primary focus of cancer research. Effective supply of $\mathrm{MKH}$, an active form of MK-4, is a promising approach concerning the suppression of HCC growth with low toxicity. MKH-DMG, a $\mathrm{MKH}$ prodrug in esterized form, can deliver substantial amounts of MKH to HCC cells, and can suppress HCC proliferation effectively both in vitro and in vivo. The anti-HCC effect of MKH-DMG may be the result of cell-cycle arrest with downregulation of NF- $\kappa \mathrm{B}$ and cyclin D1 expression. MKH-DMG is a promising new candidate for suppression of the onset of HCC, its recurrence and metastasis without significant adverse effects.

\section{Acknowledgments}

We wish to thank Naoki Magario for his assistance with the DCP assay.

\section{Author Contributions}

All authors contributed to the submitted work. Especially, ME and JT wrote and supervised the review; KM prepared the figures.

\section{Conflicts of Interest}

The authors declare no conflict of interest.

\section{References}

1. Huisse, M.G.; Leclercq, M.; Belghiti, J.; Flejou, J.F.; Suttie, J.W.; Bezeaud, A.; Stafford, D.W.; Guillin, M.C. Mechanism of the abnormal vitamin K-dependent gamma-carboxylation process in human hepatocellular carcinomas. Cancer 1994, 74, 1533-1541.

2. Li, Z.Q.; He, F.Y.; Stehle, C.J.; Wang, Z.; Kar, S.; Finn, F.M.; Carr, B.I. Vitamin K uptake in hepatocytes and hepatoma cells. Life Sci. 2002, 70, 2085-2100.

3. Orimo, H.; Shiraki, M.; Tomita, A.; Morii, H.; Fujita, T.; Ohata, M. Effects of menatetrenone on the bone and calcium metabolism in osteoporosis: A double-blind placebo-controlled study. J. Bone Miner. MeTable 1998, 16, 106-112.

4. Shiraki, M.; Shiraki, Y.; Aoki, C.; Miura, M. Vitamin K2 (menatetrenone) effectively prevents fractures and sustains lumbar bone mineral density in osteoporosis. J. Bone Miner. Res. 2000, 15, 515-521.

5. Knapen, M.H.; Schurgers, L.J.; Vermeer, C. Vitamin K2 supplementation improves hip bone geometry and bone strength indices in postmenopausal women. Osteoporos. Int. 2007, 18, 963-972.

6. Inoue, T.; Fujita, T.; Kishimoto, H.; Makino, T.; Nakamura, T.; Nakamura, T.; Sato, T.; Yamazaki, K. Randomized controlled study on the prevention of osteoporotic fractures (OF study): A phase IV clinical study of 15-mg menatetrenone capsules. J. Bone Miner. MeTable 2009, 27, 66-75. 
7. Bouzahzah, B.; Nishikawa, Y.; Simon, D.; Carr, B.I. Growth control and gene expression in a new hepatocellular carcinoma cell line, Hep40: Inhibitory actions of vitamin K. J. Cell. Physiol. 1995, $165,459-467$.

8. Wang, Z.; Wang, M.; Finn, F.; Carr, B.I. The growth inhibitory effects of vitamins K and their actions on gene expression. Hepatology 1995, 22, 876-882.

9. Otsuka, M.; Kato, N.; Shao, R.X.; Hoshida, Y.; Ijichi, H.; Koike, Y.; Taniguchi, H.; Moriyama, M.; Shiratori, Y.; Kawabe, T.; et al. Vitamin K2 inhibits the growth and invasiveness of hepatocellular carcinoma cells via protein kinase A activation. Hepatology 2004, 40, 243-251.

10. Ozaki, I.; Zhang, H.; Mizuta, T.; Ide, Y.; Eguchi, Y.; Yasutake, T.; Sakamaki, T.; Pestell, R.G.; Yamamoto, K. Menatetrenone, a vitamin K2 analogue, inhibits hepatocellular carcinoma cell growth by suppressing cyclin D1 expression through inhibition of nuclear factor kappaB activation. Clin. Cancer Res. 2007, 13, 2236-2245.

11. Kaneda, M.; Zhang, D.; Bhattacharjee, R.; Nakahama, K.; Arii, S.; Morita, I. Vitamin K2 suppresses malignancy of $\mathrm{HuH7}$ hepatoma cells via inhibition of connexin 43. Cancer Lett. 2008, 263, 53-60.

12. Hitomi, M.; Yokoyama, F.; Kita, Y.; Nonomura, T.; Masaki, T.; Yoshiji, H.; Inoue, H.; Kinekawa, F.; Kurokohchi, K.; Uchida, N.; et al. Antitumor effects of vitamins K1, K2 and K3 on hepatocellular carcinoma in vitro and in vivo. Int. J. Oncol. 2005, 26, 713-720.

13. Habu, D.; Shiomi, S.; Tamori, A.; Takeda, T.; Tanaka, T.; Kubo, S.; Nishiguchi, S. Role of vitamin $\mathrm{K} 2$ in the development of hepatocellular carcinoma in women with viral cirrhosis of the liver. Jama 2004, 292, 358-361.

14. Mizuta, T.; Ozaki, I.; Eguchi, Y.; Yasutake, T.; Kawazoe, S.; Fujimoto, K.; Yamamoto, K. The effect of menatetrenone, a vitamin K2 analog, on disease recurrence and survival in patients with hepatocellular carcinoma after curative treatment: A pilot study. Cancer 2006, 106, 867-872.

15. Yoshida, H.; Shiratori, Y.; Kudo, M.; Shiina, S.; Mizuta, T.; Kojiro, M.; Yamamoto, K.; Koike, Y.; Saito, K.; Koyanagi, N.; et al. Effect of vitamin K2 on the recurrence of hepatocellular carcinoma. Hepatology 2011, 54, 532-540.

16. Riaz, I.B.; Riaz, H.; Riaz, T.; Rahman, S.; Amir, M.; Badshah, M.B.; Kazi, A.N. Role of vitamin K2 in preventing the recurrence of hepatocellular carcinoma after curative treatment: A meta-analysis of randomized controlled trials. BMC Gastroenterol. 2012, 12, doi:10.1186/1471-230X-12-170.

17. Takata, J.; Karube, Y.; Hanada, M.; Matsunaga, K.; Matsushima, Y.; Sendo, T.; Aoyama, T. Vitamin K prodrugs: 1. Synthesis of amino acid esters of menahydroquinone-4 and enzymatic reconversion to an active form. Pharm. Res. 1995, 12, 18-23.

18. Takata, J.; Karube, Y.; Hanada, M.; Matsunaga, K.; Matsushima, Y.; Sendo, T.; Oishi, R. Vitamin K prodrugs: 2. water-soluble prodrugs of menahydroquinone-4 for systemic site-specific delivery. Pharm. Res. 1995, 12, 1973-1979.

19. Setoguchi, S.; Watase, D.; Matsunaga, K.; Matsubara, M.; Kubo, Y.; Kusuda, M.; Nagata-Akaho, N.; Enjoji, M.; Nakashima, M.; Takeshita, M.; et al. Enhanced antitumor effects of novel intracellular delivery of an active form of menaquinone-4, menahydroquinone-4, into hepatocellular carcinoma. Cancer Prev. Res. 2015, 8, 129-138.

20. Koike, Y.; Shiratori, Y.; Sato, S.; Obi, S.; Teratani, T.; Imamura, M.; Yoshida, H.; Shiina, S.; Omata, M. Des-gamma-carboxy prothrombin as a useful predisposing factor for the development of portal venous invasion in patients with hepatocellular carcinoma: A prospective analysis of 227 
patients. Cancer 2001, 91, 561-569.

21. Weitz, I.C.; Liebman, H.A. Des-gamma-carboxy (abnormal) prothrombin and hepatocellular carcinoma: A critical review. Hepatology 1993, 18, 990-997.

22. Inagaki, Y.; Tang, W.; Makuuchi, M.; Hasegawa, K.; Sugawara, Y.; Kokudo, N. Clinical and molecular insights into the hepatocellular carcinoma tumour marker des-gamma-carboxyprothrombin. Liver Int. 2011, 31, 22-35.

23. Suzuki, M.; Shiraha, H.; Fujikawa, T.; Takaoka, N.; Ueda, N.; Nakanishi, Y.; Koike, K.; Takaki, A.; Shiratori, Y. Des-gamma-carboxy prothrombin is a potential autologous growth factor for hepatocellular carcinoma. J. Biol. Chem. 2005, 280, 6409-6415.

24. Ma, M.; Qu, X.J.; Mu, G.Y.; Chen, M.H.; Cheng, Y.N.; Kokudo, N.; Tang, W.; Cui, S.X. Vitamin K2 inhibits the growth of hepatocellular carcinoma via decrease of des-gamma-carboxy prothrombin. Chemotherapy 2009, 55, 28-35.

25. Yue, P.; Gao, Z.H.; Xue, X.; Cui, S.X.; Zhao, C.R.; Yuan, Y.; Yin, Z.; Inagaki, Y.; Kokudo, N.; Tang, W.; et al. Des-gamma-carboxyl prothrombin induces matrix metalloproteinase activity in hepatocellular carcinoma cells by involving the ERK1/2 MAPK signalling pathway. Eur. J. Cancer 2011, 47, 1115-1124.

26. Matsubara, M.; Shiraha, H.; Kataoka, J.; Iwamuro, M.; Horiguchi, S.; Nishina, S.; Takaoka, N.; Uemura, M.; Takaki, A.; Nakamura, S.; et al. Des-gamma-carboxyl prothrombin is associated with tumor angiogenesis in hepatocellular carcinoma. J. Gastroenterol. Hepatol. 2012, 27, 1602-1608.

27. Gao, J.J.; Feng, X.B.; Inagaki, Y.; Song, P.P.; Kokudo, N.; Hasegawa, K.; Sugawara, Y.; Tang, W. Des-gamma-carboxy prothrombin and c-Met were concurrently and extensively expressed in hepatocellular carcinoma and associated with tumor recurrence. Biosci. Trends 2012, 6, 153-159.

28. Okuda, H; Obata, H.; Nakanishi, T.; Furukawa, R.; Hashimoto, E. Production of abnormal prothrombin (des-gamma-carboxy prothrombin) by hepatocellular carcinoma. A clinical and experimental study. J. Hepatol. 1987, 4, 357-363.

29. Furukawa, M.; Nakanishi, T.; Okuda, H.; Ishida, S.; Obata, H. Changes of plasma des-gamma-carboxy prothrombin levels in patients with hepatocellular carcinoma in response to vitamin K. Cancer $\mathbf{1 9 9 2 ,}$ 69, 31-38.

30. Murata, K.; Suzuki, H.; Okano, H.; Oyamada, T.; Yasuda, Y.; Sakamoto, A. Cytoskeletal changes during epithelial-to-fibroblastoid conversion as a crucial mechanism of des-gamma-carboxy prothrombin production in hepatocellular carcinoma. Int. J. Oncol. 2009, 35, 1005-1014.

31. Binkley, N.C.; Suttie, J.W. Vitamin K nutrition and osteoporosis. J. Nutr. 1995, 125, 1812-1821.

32. Furie, B.; Bouchard, B.A.; Furie, B.C. Vitamin K-dependent biosynthesis of gamma-carboxyglutamic acid. Blood 1999, 93, 1798-1808.

33. Stafford, D.W. The vitamin K cycle. J. Thromb. Haemost. 2005, 3, 1873-1878.

34. Hallgren, K.W.; Qian, W.; Yakubenko, A.V.; Runge, K.W.; Berkner, K.L. r-VKORC1 expression in factor IX BHK cells increases the extent of factor IX carboxylation but is limited by saturation of another carboxylation component or by a shift in the rate-limiting step. Biochemistry 2006, 45, 5587-5598.

35. Westhofen, P.; Watzka, M.; Marinova, M.; Hass, M.; Kirfel, G.; Müller, J.; Bevans, C.G.; Müller, C.R.; Oldenburg, J. Human vitamin K 2,3-epoxide reductase complex subunit 1-like 1 (VKORC1L1) 
mediates vitamin K-dependent intracellular antioxidant function. J. Biol. Chem. 2011, 286, 15085-15094.

36. Ishii, M.; Shimomura, M.; Hasegawa, J.; Asano, Y.; Yamato, T.; Yamano, Y.; Kayano, M.; Kanemaru, M.; Nakashima, M. Multiple Dose Pharmacokinetic Study of Soft Gelatin Capsule of Menatetrenone (Ea-0167) in Elderly and Young Volunteers. Jpn. Pharmacol. Ther. 1995, 23, 2637-2642.

37. Sano, Y.; Tadano, K.; Kaneko, K.; Kikuchi, K.; Yuzuriha, T. Metabolic Fate of Menatetrenone in Rats: Absorption, Distribution, Metabolism and Excretion after a Single Oral Administration. Jpn. Pharmacol. Ther. 1995, 23, 2659-2667.

38. Karin, M.; Lin, A. NF-kappaB at the crossroads of life and death. Nat. Immunol. 2002, 3, 221-227.

39. Kim, H.J.; Hawke, N.; Baldwin, A.S. NF-kappaB and IKK as therapeutic targets in cancer. Cell Death Differ. 2006, 13, 738-47.

40. Li, Z.; Wang, C.; Prendergast, G.C.; Pestell, R.G. Cyclin D1 functions in cell migration. Cell Cycle 2006, 5, 2440-2442.

(C) 2015 by the authors; licensee MDPI, Basel, Switzerland. This article is an open access article distributed under the terms and conditions of the Creative Commons Attribution license (http://creativecommons.org/licenses/by/4.0/). 